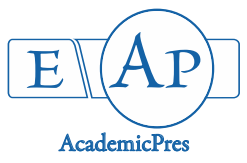

Ahmodu PB et al. (2020)

Notulae Scientia Biologicae 12(3):658-672

DOI: $10.15835 / \mathrm{nsb} 12310731$

Research Article

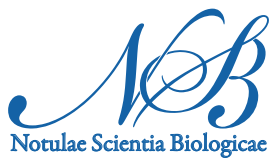

\title{
Determination of the relatedness, ease of hybridization and gene exchange among members of the genus Capsicum in Nigeria
}

\author{
Paul B. AHMODU*, Joseph A. MORAKINYO, \\ Catherine T.AHMODU \\ University of Ilorin, Faculty of Life Sciences, Department of Plant Science, Ilorin, Kwara State, \\ Nigeria; babs.paul@live.com (*corresponding author); jamorakinyo@gmail.com; ptosin49@gmail.com
}

\begin{abstract}
The fruit of Capsicum plants have a variety of names depending on place and type. This research was focused at studying the exchange of genes amongst members of the genus Capsicum in Nigeria. The process of interspecific and intervarietal hybridization of two (2) species of the genus Capsicum; C. annuum and C. frutasense were carried out and the results show that most of the studied attributes like arrangement of leaves, shape of leaves, leaf density were undetermined while majority with distinct changes follow maternal inheritance. There were less successes among the interspecific crosses and high successes between varietal crosses ranging from $19.5 \%$ to $2.4 \%$ for the inter-varietal cross and $9.3 \%$ to $2.4 \%$ for interspecific crosses. This indicates that there are more hybridization barriers among interspecific hybridization than inter-varietal hybridization. The closely related species and varieties had higher percentage success of hybridization and vice versa. Characters such as Hypocotyl colour had partial dominance. Erect flower position is dominant in W and hybrid $\mathrm{T}^{*} \mathrm{~W}$ while pendant is recessive. Red fruit colour at maturity, green fruit stalk colour was dominant while yellow was recessive. A good knowledge of how related species of the genus Capsicum are, the easier it will enable researchers to hybridize and improve the genus.
\end{abstract}

Keywords: Capsicum; crosses; dominance; exchange of genes; hybridization; maternal inheritance; recessive

\section{Introduction}

Capsicum commonly known as peppers is a genus of flowering plants in the nightshade family Solanaceae, which includes tomato, potato, tobacco, and petunia. According to International Institute of Tropical Agriculture (IITA, 2005), in Nigeria peppers are used in stew and some local dishes all over the country although the types and quantities utilized vary in different areas. The following varieties are widely grown in this country. Bird peppers 'Atawere' (Capsicum frutescens), Cayenne pepper or red pepper 'Sombo' (Capsicum frutescens), 'Atarodo' (Capsicum annum), 'Tatase' (Capsicum annum). The fruits of these types of pepper vary in size, color, shape, and pungency. They are all hot to varying degrees. Capsicum frutescens is generally hotter than Capsicum annum and there is variation within each species in terms of hotness, fruit size 
and shape. These variations suggest natural exchange of genes, at least within each species which can be determined through artificial intra specific and inter specific hybridization.

Interspecific hybridizations allow a transfer of specific genes of interest between different species, most often those involved in disease resistance (Bosland and Votava, 2000), allowing breeders to develop genetically superior genotypes. However, for the good success of such a transfer, the species must be genetically close or related, minimizing problems of incompatibility and thus enabling hybridization. As a general rule, the closer the species involved in the cross are genetically, the easier it will be to breed hybrids and the more fertile the progenies (Singh, 2002). Thus, the existence of barriers in interspecific crosses indicates the genetic distance of species and probable chromosome pairing problems within the genus. This necessitated this research in order to; determine the mode of inheritance among varieties of the genus Capsicum annum and Capsicum frutesence in Nigeria.

\section{Materials and Methods}

The study was carried out at University of Ilorin (UNILORIN) Botanical Garden between June, 2015 and December 2017. The UNILORIN Botanical Garden lies between latitude $8^{\circ} 30^{\prime} \mathrm{N}$ and longitude $4^{\circ} 33^{\prime} \mathrm{E}$ latitude $8.50^{\circ} \mathrm{N}$ and $4.550^{\circ} \mathrm{E}$, Ilorin, Kwara State, Nigeria. The first planting was done between June and November 2015 for the crosses of the parent plants to get the first generation $\left(F_{1}\right)$ seeds while the first generation $\left(F_{1}\right)$ seeds were planted between April and August, 2017 to get the second generation $\left(F_{2}\right)$ seeds, and the $\mathrm{F}_{2}$ generation were planted between September to December, 2017.

Dried seeds of three (3) varieties of Capsicum annum (varieties namely, abbreviatum ('Rodo'), grossom ('Tatase') and acumunatum ('Shombo') and Capsicum frutescence (varieties namely, baccatum ('wewe') were collected. Seven seeds of various varieties of Capsicum were obtained from local markets and were used as the parent lines. The varieties are 3 varieties of Ata of Rodo (Capsicum. annuum var. abbreviatum), Tatase ( $C$. annuum var. grossum), Shombo (C. annuum var. acuminatum), Ata wewe (C. frutascens var. baccatum), Ata Jere (C. frutascens var. baccatum). Each variety on ridges with a dimension of 30 feet $* 20$ feet. The seeds were sown in a nursery and the seedlings were transplanted with uniform spacing of 1 foot each. All varieties are sown same day; 22nd June, 2015. The seedlings were transplanted to cultivated ridges on 20th July, 2015. Plants were characterized using pepper descriptors at maturity. Single crosses and reciprocal crosses were carried out to attain the F1 seeds from the parent plants based on $n(n-1) / 2$, when $n=4$, Single and reciprocal crosses $=12$. A total of 6 single crosses and 6 reciprocal crosses. Crosses of flowers were done at the early hours of the day at about $6.00 \mathrm{am}$ to $9.00 \mathrm{am}$ before the sunrise each day before the flower opens fully and get selfed by insects and other agents of pollination. Crosses were also done in the late hours of the day for flowers just before anthesis but mature. This was done at sunset, around $6.00 \mathrm{pm}$. Cross pollinated flowers were tagged accordingly with codes such as $\mathrm{R}^{*} \mathrm{~T}$ (being Rodo*Tatase) with rodo $(\mathrm{R})$ as the female plant while Tatase $(\mathrm{T})$ as the male plant and bagged using tissue papers to avoid pollination by insects, wind or other pollinating agents.

Crosses (hybridization) were made among all four (4) in all possible combinations by physical or hand emasculation and pollination. Emasculation is the removal of stamen or anthers or killing the pollen grains of a flower without affecting the female reproductive organs and prevent self-fertilization. Pepper flower, being bisexual, hand emasculation was used. The stamens were removed using forceps. The corolla and other parts of the flower covering the androecium were cut off using scissors and proper care was taken to avoid damaging the gynoecium. Mature and viable pollen were freshly collected from the male parent plants and scooped to the mature receptive stigma of the female plant after the anther surrounding the stigma have been carefully removed. The crossed pollinated flowers were emasculated and bagged with tissue paper to ensure fertilization as crossed. Bagging was done immediately after emasculation using tissue paper bags to enclose the emasculated flowers to prevent random cross pollination; the bags are tied to the base of the flower or the flower stalk with threads. The choice of using tissue paper as bagging material was to reduce moisture and temperature inside the 
bag, which might promote fungi growth and the bags are removed within a day or two at most after pollination, during which fertilization might have taken place and fruit formation. Tagging was done immediately after bagging.

\section{Results}

Table 1 shows the percentage and successes of the parent plant crosses. Twelve (12) possible combinations were made, all were successful. It was observed that the crosses between $\mathrm{R} \times \mathrm{S}, 12$ were successful with a percentage success of $25.53 \% .27 .45 \%$ successes were observed for $\mathrm{R} \times \mathrm{T}$, with a total cross of 51 and successes of 14 . in $\mathrm{R} \times \mathrm{W}$, it was observed that 89 crosses were made, 42 were successful, 47 got aborted with a percentage success of $47.19 \%$. $S \times \mathrm{R}$ had a percentage success of $37.14 \%$, a total of 70 crosses was done for the combination, 26 were successful and 44 were aborted. In $S \times \mathrm{T}$ combination, 33 crosses were done, 18 were successful, giving a percentage success of $54.55 \% .40$ crosses were observed for $S \times W, 17$ of it were successful, 23 were failed crosses, giving a percentage success of $42.50 \%$. The cross $\mathrm{T} \times \mathrm{R}$ had 11 successes out of 58 crosses with a percentage success of $18.97 \%$, while $\mathrm{T} \times \mathrm{S}$ had 19 successes out of 30 crosses with a percentage success of $63.33 \%$. The cross $\mathrm{T} \times \mathrm{W}$ had 9 successes out of 29 crosses with a percentage success of $31.03 \%$, while $\mathrm{W} \times \mathrm{R}$ had 38 successes out of 115 crosses with a percentage success of $33.04 \%$. The crosses between $\mathrm{W} \times \mathrm{S}$ had 10 successes out of 33 crosses with a percentage success of $30.30 \%$ while $\mathrm{W} \times \mathrm{T}$ had 26 successes out of 46 crosses and a percentage success of $56.52 \%$.

Genetic exchange of characters was noticed during the crosses. Gene exchange between groups or individual organisms can be influenced by several factors which may include physical barriers against cross pollination. Such barriers are overcome through artificial land pollination. However, there are other postpollination barriers which artificial land pollination cannot remove. In all cases the effectiveness of these barriers depends on the genetic relationship between individuals and groups.

From the quantitative data of hybrids and reciprocals studied in the research. The hybrids and reciprocals exhibited segregation of characters in the $F_{1}$ and independent assortment of characters in $F_{2} 9$ quantitative characters were studied and mean values were analyzed using analysis of variance (ANNOVA) and the Duncan of plant height (PH), leaf length (LL), leaf breadth (LB), stem girth (SG), laminar leaf length (LLL), anthal length (AL), style length (SL), petal length (PL) and petal width (PW).

The variation in $\mathrm{PH}$ between the varieties and hybrids show that the different varieties of pepper have varying gene alleles for the character. There was drastic increase in variation of $\mathrm{PH}$ in the hybrid $\mathrm{R}^{*} \mathrm{~W}_{2}$ (36.20 \pm 4.39$)$ which is an increase from the hybrid parents $\mathrm{R}(27.22 \pm 3.62)$ and $\mathrm{W}(39.30 \pm 2.21)$. There was a decrease in the $\mathrm{PH}$ of hybrid $\mathrm{S}^{*} \mathrm{~W}(35.86 \pm 0.80)$ compared to the variation in $\mathrm{PH}$ of parent plants $S$ $(35.20 \pm 1.48)$ and $\mathrm{W}(39.30 \pm 2.21)$.

There was variation in LL among the hybrids. Notably in $\mathrm{W}^{*} \mathrm{R}(7.80 \pm 0.29)$ relative the parent plants $\mathrm{W}(12.64 \pm 0.92)$ and $\mathrm{R}(17.14 \pm 0.71)$. This was also noted in $S^{*} \mathrm{~T}(8.06 \pm 0.31)$ from the parents $S(13.30 \pm 0.45)$ and $\mathrm{T}(15.30 \pm 0.45)$. This can be attributed to variation in alleles of the genes responsible for LL between parents. No dominance nor recessiveness of this trait among the parents.

With hybridization there was an increase in SG in the hybrid offspring of the cross. T*R, T*W, W* $S$ and $S^{*} T$ had drastic increase in SG. There was variation of LLL among hybrid plants and their parents. This can be attributed to the random pairing of the alleles of the genes that regulate these characters.

Majorities of the data followed maternal inheritance. This can be found in tables 9 to 20. While few amongst the characters followed paternal inheritance. Majority of the characters could not be determined since both parents showed similar attributes for the same character. Examples of these characters are, calyx pigmentation, Leaf type, Leaf venation, leaf arrangement, e.t.c.

From plate 2, it was noticed that hybrid fruits are smaller in size in size when compared to their parent fruits. About $70 \%$ of the fruits were discovered to have shrinked. 
Based on interspecific crosses, it was seen that crosses between $C$. frutasense ${ }^{*} C$. annuum are higher at an average percentage than crosses between $C$. annuum and $C$. frutasence. Both at an average percentage. This means reciprocal successes are few.

Most plants crossed with W at their orientation of flower and fruits showed alternation of the character of flower orientation as some plants had either erect flowers like W while others had pendant flowers. Plants crossed with Whad similar erect flowers which were not shown in W parent plants and this implies that erect flower orientation could be said to be coded by a heterogenous pair of chromosomes. This is found in table 15 . Similar result was observed in the work done by Nwankiti (1976); and Odland and Porter (1941).

Table 1. Percentage cross pollination success among varieties of Capsicum sp.

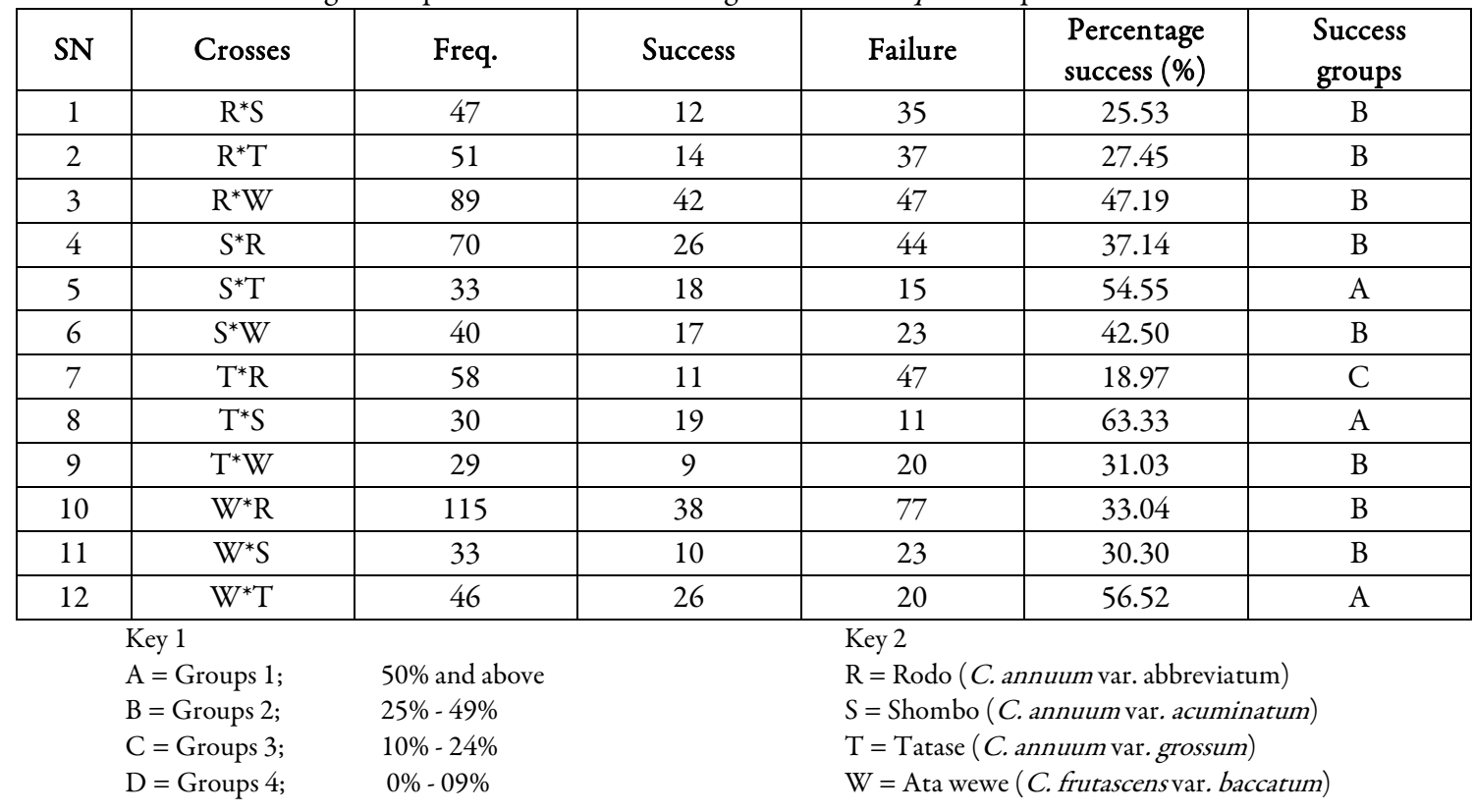

Table 2. Percentage success group

\begin{tabular}{|c|c|c|c|c|c|}
\hline S/N & Group & $\begin{array}{c}\text { C. annuum } x \\
\text { C.annuum }\end{array}$ & $\begin{array}{c}\text { C. annuum } x \\
\text { C. frutescens }\end{array}$ & $\begin{array}{c}\text { C. frutescens } x \\
\text { C. frutescens }\end{array}$ & $\begin{array}{c}\text { C. frutescens } x \\
\text { C. annuum }\end{array}$ \\
\hline 1 & $\mathrm{~A}(50 \%<)$ & 19.5 & 7.3 & 2.4 & 4.9 \\
\hline 2 & $\mathrm{~B}(25-49 \%)$ & 14.6 & 12.2 & & 14.6 \\
\hline 3 & $\mathrm{C}(10-24 \%)$ & 14.6 & 2.4 & & 2.4 \\
\hline 4 & $\mathrm{D}(0-9 \%)$ & 2.4 & & & 2.4 \\
\hline
\end{tabular}

The different groups, i.e. 1-4 denotes the grouping of the different percentage successes of hybridization from the crosses 

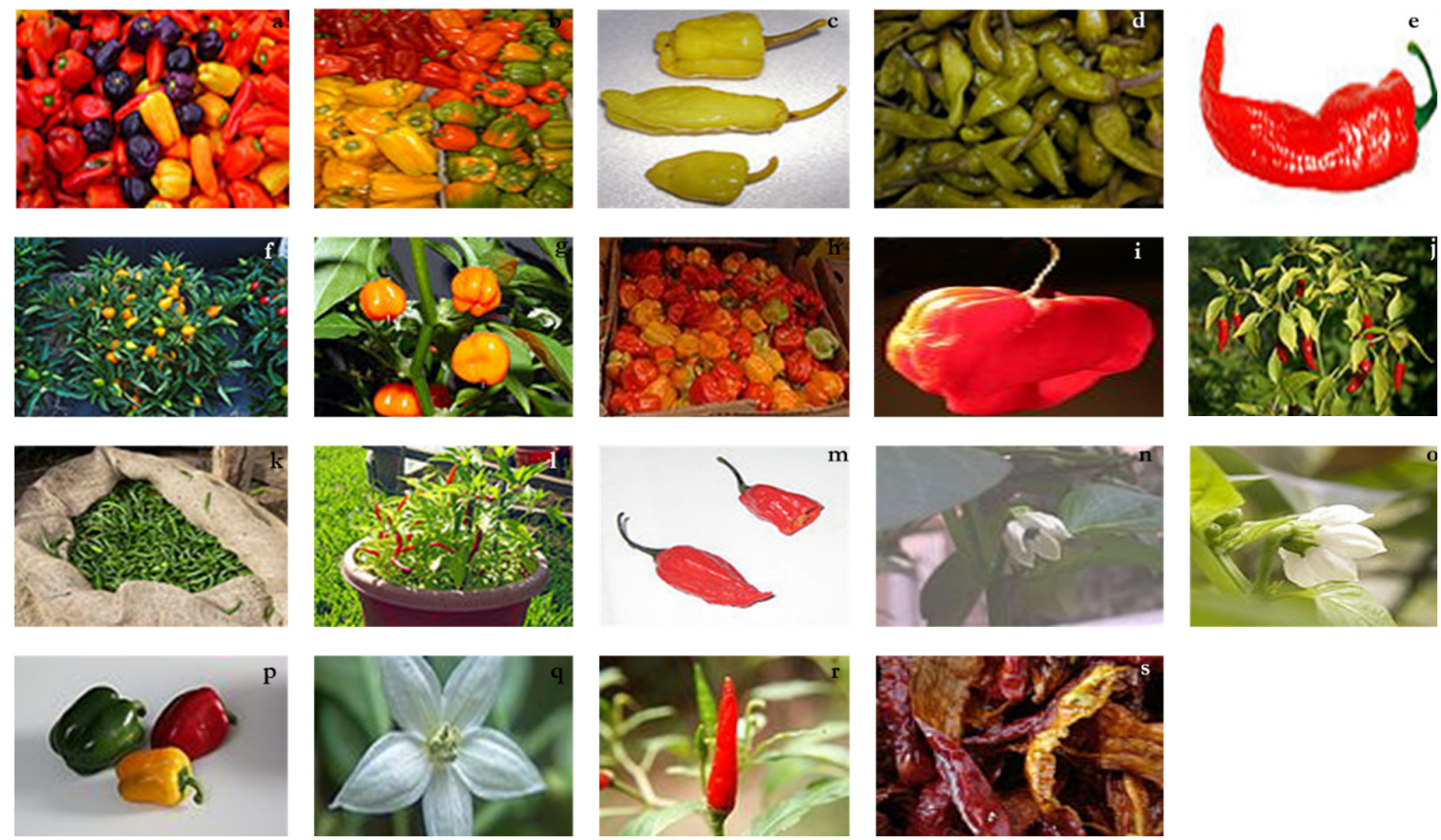

Figure 1. C. annuum cultivars

a: A variety of coloured Capsicum; b: Peperoncini (C. annuum); c: Peperoncini in kebab restaurant; d: Cayenne pepper (C. annuum); e: Compact plant of orange Capsicum; f: Habanero chili (C. chinense Jacquin) - plant with flower and fruit; g: Scotch bonnet (C. chinense) in a Caribbean market; h: Scotch bonnet; i: Thai peppers (C. annuum); j: Fresh Indian green chillies in Bangalore market; k: Piri piri (C. frutescens African Devil'); 1: Naga jolokia pepper (bhut jolokia) (C. chinensex $C$. frutescens); m: $C$. annuum flower; n: $C$. annum flower close-up; o: Green, yellow, and red peppers; p: The flower of red hot bangi pepper, Malaysia; q: A small but very hot Capsicum in Malaysia; r: Dried and crunchy Capsicum from Basilicata; s: Capsicum in Bangladesh

Source: https://en.wikipedia.org
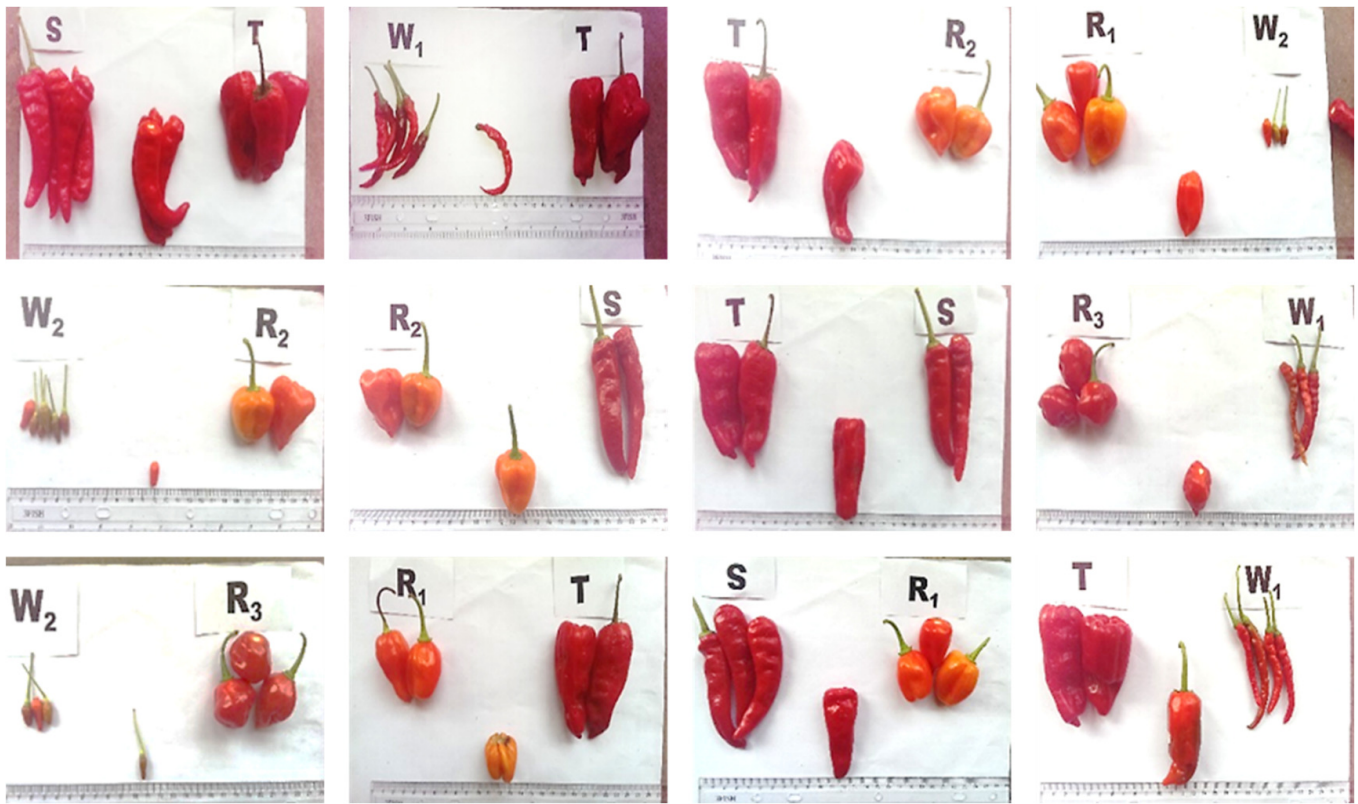

Figure 2. $\mathrm{F}_{0}$ hybrid fruits 
Ahmodu PB et al. (2020). Not Sci Biol 12(3):658-672

Table 3. Means and standard deviation of quantitative characters in all the parents and their crosses

\begin{tabular}{|c|c|c|c|c|c|c|c|c|c|}
\hline $\begin{array}{l}\text { Varieties } \\
\text { and } \\
\text { hybrids }\end{array}$ & $\begin{array}{l}\text { Plant } \\
\text { height }\end{array}$ & $\begin{array}{l}\text { Leaf } \\
\text { length }\end{array}$ & $\begin{array}{c}\text { Leaf } \\
\text { breadth }\end{array}$ & $\begin{array}{l}\text { Stem } \\
\text { girth }\end{array}$ & $\begin{array}{l}\text { Laminar leaf } \\
\text { length }\end{array}$ & $\begin{array}{l}\text { Anthal } \\
\text { length }\end{array}$ & $\begin{array}{l}\text { Style } \\
\text { length }\end{array}$ & $\begin{array}{l}\text { Petal } \\
\text { length }\end{array}$ & $\begin{array}{l}\text { Petal } \\
\text { width }\end{array}$ \\
\hline $\mathrm{R}$ & $27.22 \pm 3.62^{\text {bed }}$ & $17.14 \pm 0.71^{\text {gh }}$ & $5.80 \pm 0.40^{\text {ghij }}$ & $1.02 \pm 0.22^{\mathrm{a}}$ & $10.12 \pm 1.76^{\mathrm{bcdc}}$ & $0.24 \pm 0.19^{\mathrm{a}}$ & $0.32 \pm 0.01^{\mathrm{a}}$ & $0.80 \pm 0.03^{g}$ & $0.31 \pm 0.00^{\mathrm{ab}}$ \\
\hline$S$ & $35.20 \pm 1.48^{\text {fgh }}$ & $13.30 \pm 0.45^{\text {def }}$ & $4.30 \pm 0.07^{\mathrm{cdc}}$ & $0.60 \pm 0.09^{a}$ & $9.64 \pm 0.31^{\text {bed }}$ & $0.28 \pm 0.14^{\mathrm{b}}$ & $0.51 \pm 0.01^{\text {ef }}$ & $0.84 \pm 0.02^{g}$ & $0.72 \pm 0.01^{\mathrm{h}}$ \\
\hline $\mathrm{T}$ & $41.48 \pm 1.82^{\text {ghi }}$ & $15.3 \pm 0.45^{\text {cf }}$ & $4.65 \pm 0.09^{\text {cdefg }}$ & $0.86 \pm 0.09^{a}$ & $10.58 \pm 0.30^{\text {bcdef }}$ & $0.31 \pm 0.06^{\mathrm{bc}}$ & $0.42 \pm 0.01^{c}$ & $1.14 \pm 0.04^{\mathrm{i}}$ & $0.51 \pm 0.00^{g}$ \\
\hline W & $39.30 \pm 2.21^{\text {fgh }}$ & $12.64 \pm 0.92^{\mathrm{cde}}$ & $2.74 \pm 0.06^{\mathrm{a}}$ & $0.98 \pm 0.12^{\mathrm{a}}$ & $7.52 \pm 0.85^{\mathrm{ab}}$ & $0.31 \pm 0.00^{\mathrm{bc}}$ & $0.51 \pm 0.01^{\text {cf }}$ & $0.96 \pm 0.19^{h}$ & $0.41 \pm 0.00^{\text {ef }}$ \\
\hline$\overline{\mathrm{R}^{*} \mathrm{~W}}$ & $36.20 \pm 4.39^{\text {efgh }}$ & $12.52 \pm 1.63^{\mathrm{cdc}}$ & $6.30 \pm 0.90^{i j}$ & $1.84 \pm 0.16^{\mathrm{bc}}$ & $10.40 \pm 1.86^{\text {bcdef }}$ & $0.30 \pm 0.03^{b c}$ & $0.30 \pm 0.01^{a}$ & $0.87 \pm 0.18^{g}$ & $0.40 \pm 0.01^{\mathrm{ef}}$ \\
\hline $\mathrm{R}^{*} \mathrm{~S}$ & $33.34 \pm 1.84^{\mathrm{defg}}$ & $15.72 \pm 1.29^{\text {fgh }}$ & $9.460 .35^{\mathrm{k}}$ & $1.64 \pm 0.40^{b}$ & $13.18 \pm 1.33^{\mathrm{f}}$ & $0.31 \pm 0.00^{\mathrm{bc}}$ & $0.46 \pm 0.03^{\mathrm{cdc}}$ & $0.67 \pm 0.03^{\text {def }}$ & $0.36 \pm 0.02^{\text {cd }}$ \\
\hline $\mathrm{R}^{*} \mathrm{~T}$ & $21.86 \pm 2.21^{\mathrm{ab}}$ & $12.74 \pm 1.42^{\mathrm{de}}$ & $5.32 \pm 0.56^{\text {efghhij }}$ & $1.96 \pm 0.39^{\mathrm{bcd}}$ & $8.64 \pm 1.10^{\mathrm{b}}$ & $0.30 \pm 0.00^{\mathrm{bc}}$ & $0.48 \pm 0.02^{\mathrm{def}}$ & $0.64 \pm 0.04^{\text {cde }}$ & $0.35 \pm 0.02^{\mathrm{cd}}$ \\
\hline $\mathrm{S}^{*} \mathrm{R}$ & $36.88 \pm 3.28^{\text {efgh }}$ & $11.20 \pm 1.62^{\text {bce }}$ & $4.28 \pm 0.33^{\mathrm{cdc}}$ & $1.90 \pm 0.14^{\mathrm{bc}}$ & $8.84 \pm 0.21^{b}$ & $0.30 \pm 0.01^{\mathrm{bc}}$ & $0.52 \pm 0.10^{\text {cf }}$ & $0.54 \pm 0.02^{\mathrm{ab}}$ & $0.31 \pm 0.00^{\mathrm{ab}}$ \\
\hline$S^{*} \mathrm{~T}$ & $42.24 \pm 3.29^{\mathrm{hi}}$ & $8.06 \pm 0.31^{a}$ & $3.52 \pm 0.28^{\text {abc }}$ & $3.32 \pm 0.42^{f}$ & $5.08 \pm 0.29^{\mathrm{a}}$ & $0.40 \pm 0.00^{c}$ & $0.52 \pm 0.04^{\mathrm{f}}$ & $0.84 \pm 0.04^{g}$ & $0.44 \pm 0.02^{\mathrm{f}}$ \\
\hline$S^{*} \mathrm{~W}$ & $35.86 \pm 0.80^{\text {efgh }}$ & $12.02 \pm 1.05^{\text {bcd }}$ & $4.38 \pm 0.49^{\text {cdef }}$ & $2.74 \pm 0.35^{\mathrm{e}}$ & $10.31 \pm 0.99^{\text {bcdef }}$ & $0.41 \pm 0.01^{e}$ & $0.60 \pm 0.00^{\mathrm{g}}$ & $0.80 \pm 0.01^{g}$ & $0.30 \pm 0.00^{\mathrm{a}}$ \\
\hline $\mathrm{T}^{*} \mathrm{R}$ & $18.62 \pm 2.09^{\mathrm{a}}$ & $13.98 \pm 0.70^{\text {def }}$ & $3.86 \pm 0.43^{\text {abcd }}$ & $1.84 \pm 0.23^{\mathrm{bc}}$ & $12.76 \pm 0.80^{\text {ef }}$ & $0.31 \pm 0.01^{\mathrm{bc}}$ & $0.33 \pm 0.03^{\mathrm{ab}}$ & $0.57 \pm 0.02^{\mathrm{bc}}$ & $0.31 \pm 0.01^{\mathrm{ab}}$ \\
\hline $\mathrm{T}^{*} \mathrm{~W}$ & $35.88 \pm 0.92^{\text {efgh }}$ & $12.04 \pm 0.71^{\text {bcd }}$ & $4.36 \pm 0.19^{\text {cdef }}$ & $3.92 \pm 0.10^{f}$ & $9.48 \pm 0.58^{\mathrm{bc}}$ & $0.32 \pm 0.20^{c}$ & $0.40 \pm 0.10^{\mathrm{bc}}$ & $0.49 \pm 0.01^{a}$ & $0.41 \pm 0.01^{\mathrm{cf}}$ \\
\hline $\mathrm{W}^{*} \mathrm{R}$ & $38.94 \pm 0.97^{\text {fgh }}$ & $7.80 \pm 0.29^{\mathrm{a}}$ & $2.92 \pm 0.09^{\mathrm{ab}}$ & $2.46 \pm 0.16^{\mathrm{cde}}$ & $4.88 \pm 0.20^{\mathrm{a}}$ & $0.30 \pm 0.01^{\mathrm{bc}}$ & $0.39 \pm 0.01^{\mathrm{bc}}$ & $0.72 \pm 0.01^{\text {cd }}$ & $0.34 \pm 0.02^{\mathrm{bc}}$ \\
\hline \multirow[t]{2}{*}{$\mathrm{W}^{*} \mathrm{~S}$} & $36.58 \pm 0.86^{\text {efgh }}$ & $11.18 \pm 0.30^{\text {bed }}$ & $4.72 \pm 0.10^{\text {cdefg }}$ & $3.44 \pm 0.02^{\mathrm{f}}$ & $7.56 \pm 0.24^{\mathrm{ab}}$ & $0.40 \pm 0.01^{c}$ & $0.60 \pm 0.03^{g}$ & $0.80 \pm 0.03^{g}$ & $0.40 \pm 0.01^{\mathrm{e}}$ \\
\hline & $\mathrm{S}$ & $\mathrm{S}$ & $\mathrm{S}$ & $\mathrm{S}$ & $\mathrm{S}$ & $\mathrm{S}$ & $\mathrm{S}$ & S & $\mathrm{S}$ \\
\hline
\end{tabular}
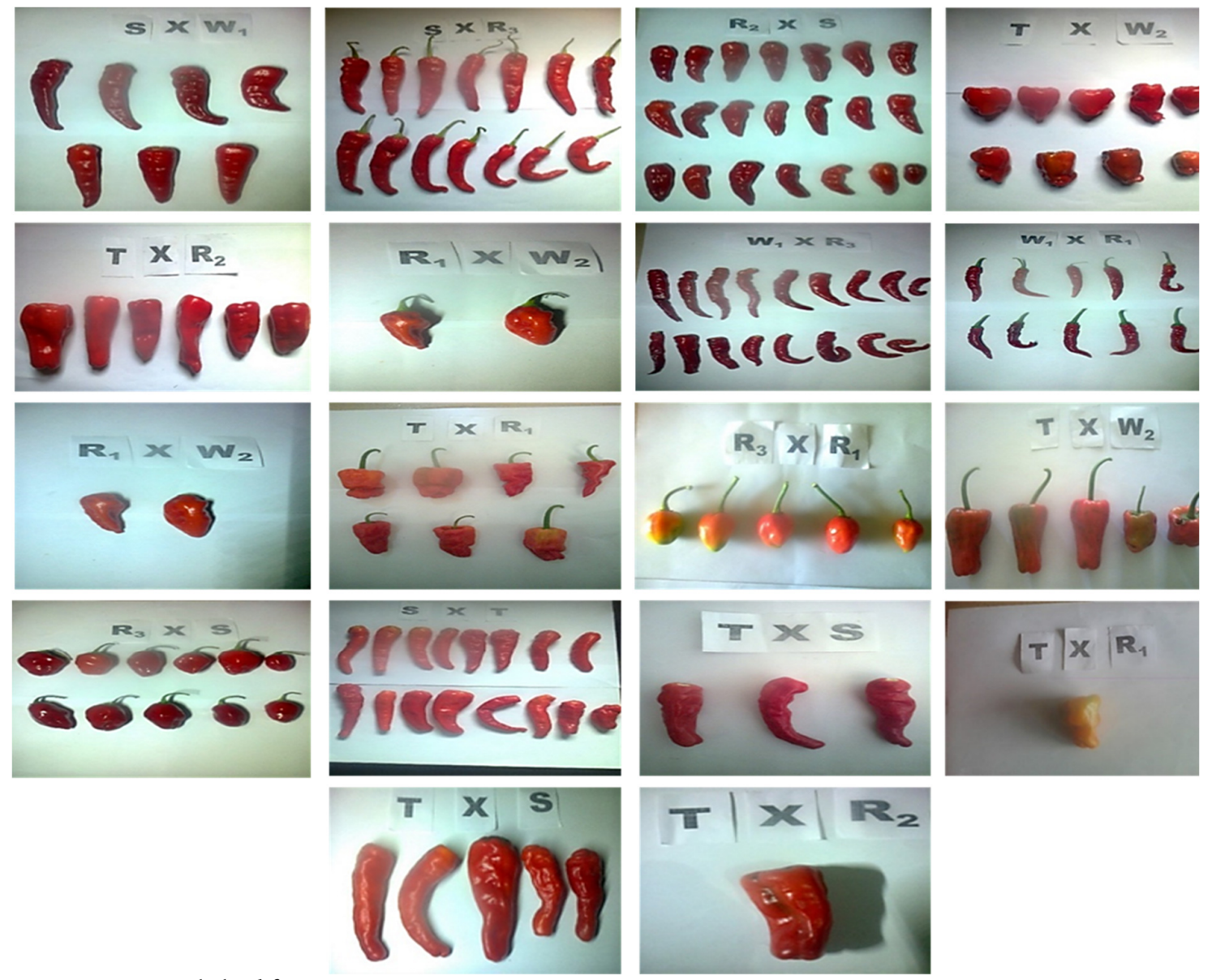

Figure 3. $F_{0}$ hybrid fruits 
Table 4. Qualitative/quantitative fruit characters of $F_{1}$ plants

\begin{tabular}{|c|c|c|c|c|c|c|}
\hline \multicolumn{7}{|c|}{ Fruit characters } \\
\hline Hybrids & $\begin{array}{l}\text { Pedicel } \\
\text { length } \\
(\mathrm{cm})\end{array}$ & $\begin{array}{l}\text { Fruit width } \\
\qquad(\mathrm{cm})\end{array}$ & $\begin{array}{l}\text { Fruit length } \\
\qquad(\mathrm{cm})\end{array}$ & $\begin{array}{c}\text { Fruit weight with } \\
\text { pedicel } \\
(\mathrm{mg})\end{array}$ & $\begin{array}{c}\text { Fruit weight } \\
\text { without } \\
\text { pedicel }(\mathrm{cm})\end{array}$ & Fruit colour \\
\hline $\mathrm{R}^{*} \mathrm{~S}$ & 3.40 & 5.65 & 5.65 & 7.30 & 7.10 & Red \\
\hline $\mathrm{R}^{*} \mathrm{~T}$ & 3.30 & 4.9 & 2.90 & 6.06 & 6.02 & Orange \\
\hline $\mathrm{R}^{*} \mathrm{~W}$ & 4.20 & 4.8 & 40.00 & 9.16 & 8.52 & Red \\
\hline $\mathrm{S}^{*} \mathrm{R}$ & 5.10 & 3.75 & 7.10 & 10.40 & 10.05 & Red \\
\hline $\mathrm{S}^{*} \mathrm{~T}$ & 3.65 & 2.4 & 11.25 & 10.61 & 10.29 & Red \\
\hline $\mathrm{S}^{*} \mathrm{~W}$ & 4.10 & 3 & 8.20 & 6.70 & 6.50 & Red \\
\hline $\mathrm{T}^{*} \mathrm{R}$ & 4.90 & 4.15 & 3.95 & 6.20 & 6.00 & Red / Variegated \\
\hline $\mathrm{T}^{*} \mathrm{~S}$ & 5.70 & 2.9 & 6.80 & 12.54 & 12.17 & Red \\
\hline $\mathrm{T}^{*} \mathrm{~W}$ & 4.55 & 4.95 & 8.55 & 10.50 & 10.30 & Red \\
\hline $\mathrm{W}^{*} \mathrm{R}$ & 3.20 & 1.6 & 7.20 & 1.30 & 1.22 & Red \\
\hline $\mathrm{W}^{*} \mathrm{~S}$ & 3.43 & 1.62 & 8.57 & 1.83 & 1.63 & Red \\
\hline $\mathrm{W}^{*} \mathrm{~T}$ & 3.90 & 1.10 & 7.97 & 1.56 & 1.44 & Red \\
\hline
\end{tabular}

Table 5. Quantitative and floral data of $F_{1}$ hybrid plants

\begin{tabular}{|c|c|c|c|c|c|c|c|c|c|c|c|c|c|c|}
\hline \multicolumn{15}{|c|}{$F_{1}$ hybrids } \\
\hline \multicolumn{6}{|c|}{ Quantitative characters } & \multicolumn{9}{|c|}{ Floral characters } \\
\hline & Flower position & $\begin{array}{c}\text { Pollen } \\
\text { sac } \\
\text { colour }\end{array}$ & $\begin{array}{l}\text { Style } \\
\text { colour }\end{array}$ & $\begin{array}{l}\text { Stigma } \\
\text { colour }\end{array}$ & $\begin{array}{c}\text { Stigma } \\
\text { position }\end{array}$ & $\begin{array}{c}\text { Flower } \\
\text { stalk } \\
\text { lenth } \\
(\mathrm{cm})\end{array}$ & $\begin{array}{c}\text { Petals } \\
\text { length } \\
(\mathrm{cm})\end{array}$ & $\begin{array}{l}\text { Calyx } \\
\text { length } \\
(\mathrm{cm})\end{array}$ & $\begin{array}{l}\text { Style } \\
\text { length } \\
(\mathrm{cm})\end{array}$ & 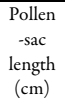 & $\begin{array}{c}\text { Leaf } \\
\text { breadth } \\
(\mathrm{cm})\end{array}$ & $\begin{array}{l}\text { Leaf } \\
\text { length } \\
(\mathrm{cm})\end{array}$ & $\begin{array}{l}\text { Stem } \\
\text { girth } \\
(\mathrm{cm})\end{array}$ & $\begin{array}{c}\text { Plant } \\
\text { height } \\
(\mathrm{cm})\end{array}$ \\
\hline$r^{*} w$ & $\begin{array}{c}\text { pendant/ } \\
\text { pointing upward }\end{array}$ & green & green & green & same level & 2.7 & 0.5 & 0.4 & 0.5 & 0.3 & 5.3 & 10.52 & 1.84 & 23.12 \\
\hline$s^{*} \mathrm{r}$ & pendant & purple & purple & $\begin{array}{l}\text { light } \\
\text { green }\end{array}$ & protruding & 2.8 & 0.5 & 0.4 & 0.5 & 0.3 & 4.28 & 9.38 & 1.9 & 24.42 \\
\hline$t^{*} r$ & pendant & purple & green & green & submerged & 2.6 & 0.6 & 0.5 & 0.34 & 0.34 & 3.86 & 11.98 & 1.84 & 16.62 \\
\hline$r^{*} s$ & $\begin{array}{c}\text { pendant / upward } \\
\text { curvy }\end{array}$ & $\begin{array}{l}\text { green/ } \\
\text { purple }\end{array}$ & green & purple & protruding & 2.8 & 0.5 & 0.4 & 0.5 & 0.32 & 9 & 18.2 & 1.7 & 25.4 \\
\hline $\mathrm{t}^{*} \mathrm{~s}$ & pendant & $\begin{array}{l}\text { green/ } \\
\text { purple }\end{array}$ & purple & green & protruding & 2.15 & 0.8 & 0.45 & 0.5 & 0.39 & 3.52 & 8.06 & 3.32 & 33.6 \\
\hline$s^{*} w$ & pendant & green & purple & green & submerged & 1.8 & 0.8 & 0.4 & 0.6 & 0.4 & 4.38 & 10.36 & 2.74 & 27.58 \\
\hline $\mathrm{r}^{*} \mathrm{t}$ & conical pendant & green & purple & green & protruding & 1.8 & 0.7 & 0.5 & 0.5 & 0.4 & 5.35 & 12.2 & 1.925 & 20.85 \\
\hline $\mathrm{w}^{*} \mathrm{r}$ & pendant & purple & green & green & $\begin{array}{l}\text { submerged/ } \\
\text { protruding }\end{array}$ & 1.8 & 0.7 & 0.38 & 0.4 & 0.3 & 2.9 & 7.51 & 2.8 & 40.9 \\
\hline $\mathrm{w}^{*} \mathrm{~s}$ & erect & purple & green & green & protruding & 0.8 & 0.5 & 0.3 & 0.4 & 0.4 & 4.4 & 10.6 & 3.4 & 35.7 \\
\hline
\end{tabular}

Table 6. Comparison of $\mathrm{R}$ and $\mathrm{T}$

\begin{tabular}{|c|c|c|c|c|c|}
\hline \multicolumn{6}{|c|}{ Determination of dominance between parents } \\
\hline $\mathrm{s} / \mathrm{n}$ & Character & Parent 1 & Parent 2 & Hybrid & $\begin{array}{l}\text { Inference on mode of } \\
\text { inheritance }\end{array}$ \\
\hline & & $\mathrm{R}$ & $\mathrm{T}$ & $\mathrm{T} \times \mathrm{R}$ & \\
\hline 1 & Stem colour & Green & Purple & Green/Purple & No dominance \\
\hline 2 & Stem pubescence & Intermediate & Sparse & Sparse & Resembles male parent \\
\hline 3 & Leaf colour & Light green & Dark green & Green & No dominance \\
\hline 4 & Fresh fruit colour & Yellow & Green & Green & Resembles male parent \\
\hline 5 & Fruit pubescence & Sparse & Sparse & Sparse & Not determined \\
\hline 6 & fruit stalk colour & Green & Green & Green & Not determined \\
\hline 7 & Days to germination & 7 & 6 & 7 & Quantitative \\
\hline 8 & Hypocotyl colour & Purple & Purple & Purple & Not determined \\
\hline 9 & Hypocotyl pubescence & Intermediate & Sparse & Sparse & Resembles female parent \\
\hline 10 & $\begin{array}{l}\text { Anthocyanin on the } \\
\text { nodes }\end{array}$ & Green & Dark purple & Green & Resembles female parent \\
\hline 11 & Stem pubescence & Dense & Sparsed & Dense & Resembles female parent \\
\hline 12 & Plant growth habit & Erect & Erect & Erect & Not determined \\
\hline
\end{tabular}


Ahmodu PB et al. (2020). Not Sci Biol 12(3):658-672

\begin{tabular}{|c|c|c|c|c|c|}
\hline 13 & Branching habit & Dense & Dense & Dense & Not determined \\
\hline 14 & Leaf density & Dense & Dense & Dense & Not determined \\
\hline 15 & Leaf colour & Green & Dark green & dark green & Resembles male parent \\
\hline 16 & Leaf venation & Parallel & Pinnate & pinnate & Not determined \\
\hline 17 & Leaf type & Compound & Compound & compound & Not determined \\
\hline 18 & Leaf arrangement & Alternate & Alternate & Alternate & Not determined \\
\hline 19 & Leaf pubescence & Intermediate & Intermediate & Intermediate & Not determined \\
\hline 20 & $\begin{array}{c}\text { Pedicel position at } \\
\text { anthesis }\end{array}$ & Intermediate & Pendant & Pendant & Resembles male parent \\
\hline 21 & $\begin{array}{c}\text { Filament colour after } \\
\text { anthesis }\end{array}$ & Green & Purple & Green & Resembles female parent \\
\hline 22 & Flower position & Pendant & Pendant & Pendant & Not determined \\
\hline 23 & Fruit shape & Sunken & Sunken & Sunken & Not determined \\
\hline 24 & $\begin{array}{c}\text { Fruit surface } \\
\text { wrinkled }\end{array}$ & Semi-wrinkled & $\begin{array}{c}\text { Semi } \\
\text { wrinkled }\end{array}$ & Semi- & Rotermined \\
\hline 25 & $\begin{array}{c}\text { Stigma position at } \\
\text { anthesis }\end{array}$ & Exserted & same level & Exserted & Resembles female parent \\
\hline 26 & Calyx pigmentation & Present & Present & Present & Not determined \\
\hline 27 & $\begin{array}{c}\text { Fruit colour at } \\
\text { immature stage }\end{array}$ & Yellow & Green & Green & Resembles male parent \\
\hline 28 & Fruit colour at maturity & Lemon & Red & Red & Resembles male parent \\
\hline 29 & Pedicel with fruit & Persistent & Persistent & Persistent & Not determined \\
\hline
\end{tabular}

Table 7. Comparison of R and W

\begin{tabular}{|c|c|c|c|c|c|}
\hline \multicolumn{6}{|c|}{ Determination of dominance between parents } \\
\hline $\mathrm{s} / \mathrm{n}$ & Character & Parent 1 & Parent 2 & Hybrid & Inference on mode of inheritance \\
\hline & & $\mathrm{R}$ & $\mathrm{W}$ & W X R & \\
\hline 1 & Stem colour & Green & Purple & Green & Resembles female parent \\
\hline 2 & Stem pubescence & Intermediate & Sparse & Sparse & Resembles male parent \\
\hline 3 & Leaf colour & Light green & Dark green & Green & Not dominance/Reccessiveness \\
\hline 4 & Fresh fruit colour & Yellow & Green & Green & Resembles male parent \\
\hline 5 & Fruit pubescence & Sparse & Non & Non & Resembles male parents \\
\hline 6 & fruit stalk colour & Green & Green & Green & Not determined \\
\hline 7 & Days to germination & 7 & 8 & 7 & Quantitative \\
\hline 8 & Hypocotyl colour & Purple & Dark green & Green & No dominance \\
\hline 9 & $\begin{array}{l}\text { Hypocotyl } \\
\text { pubescence }\end{array}$ & Intermediate & Sparse & Sparse & Resembles male parents \\
\hline 10 & $\begin{array}{l}\text { Anthocyanin on the } \\
\text { nodes }\end{array}$ & Green & Purple & Green & Resembles female parents \\
\hline 11 & Stem pubescence & Dense & Dense & Dense & Not determined \\
\hline 12 & Plant growth habit & Erect & Erect & Erect & Not determined \\
\hline 13 & Branching habit & Dense & Dense & Dense & Not determined \\
\hline 14 & Leaf density & Dense & Dense & Dense & Not determined \\
\hline 15 & Leaf colour & Green & Dark green & Green & Resembles female parents \\
\hline 16 & Leaf venation & Parallel & Parallel & Parallel & Not determined \\
\hline 17 & Leaf type & Compound & Compound & Compound & Not determined \\
\hline 18 & leaf arrangement & Alternate & Alternate & Alternate & Not determined \\
\hline 19 & Leaf pubescence & Intermediate & Intermediate & Intermediate & Not determined \\
\hline 20 & $\begin{array}{c}\text { Pedicel position at } \\
\text { anthesis }\end{array}$ & Intermediate & Pendant & Pendant & Resembles male parents \\
\hline 21 & Filament colour & Green & White & White & Resembles male parents \\
\hline
\end{tabular}


Ahmodu PB et al. (2020). Not Sci Biol 12(3):658-672

\begin{tabular}{|c|c|c|c|c|c|}
22 & Flower position & Intermediate & Pendant & Intermediate & Resembles female parents \\
\hline 23 & Fruit shape & Sunken & Elongated & elongated & Resembles female parents \\
\hline 24 & Fruit surface & $\begin{array}{c}\text { Semi- } \\
\text { wrinkled }\end{array}$ & Wrinkled & wrinkled & Resembles male parents \\
\hline 25 & $\begin{array}{c}\text { Stigma position at } \\
\text { anthesis }\end{array}$ & Exserted & Exserted & Exserted & Not determined \\
\hline 26 & $\begin{array}{c}\text { Calyx pigmentation } \\
\text { Fruit colour at }\end{array}$ & Present & Present & Present & Not determined \\
\hline 28 & $\begin{array}{c}\text { Fruit colour at } \\
\text { maturity }\end{array}$ & Red & Reen & Red & Resembles male parents \\
\hline 29 & Pedicel with fruit & Persistent & Persistent & Persistent & Not determined \\
\hline
\end{tabular}

Table 8. Comparison of $\mathrm{T}$ and $\mathrm{R}$

\begin{tabular}{|c|c|c|c|c|c|}
\hline \multicolumn{6}{|c|}{ Determination of dominance between parents } \\
\hline $\begin{array}{l}\mathrm{s} / \\
\mathrm{n}\end{array}$ & Character & Parent 1 & Parent 2 & Hybrid & Inference on mode of inheritance \\
\hline & & $\mathrm{R}$ & $\mathrm{T}$ & TXR & \\
\hline 1 & Stem colour & Green & Purple & Green/purple & No dominance \\
\hline 2 & Stem pubescence & Intermediate & Sparse & Sparse & Resembles male parent \\
\hline 3 & Leaf colour & Green & Dark green & Green & Resembles female parents \\
\hline 4 & Fresh fruit colour & Yellow & Green & Green & Resembles male parent \\
\hline 5 & Fruit pubescence & Sparse & Sparse & Sparse & Not determined \\
\hline 6 & fruit stalk colour & Green & Green & Green & Not determined \\
\hline 7 & Days to germination & 7 & 6 & 7 & Quantitative \\
\hline 8 & Hypocotyl colour & Light green & Purple & Purple/green & No dominance \\
\hline 9 & $\begin{array}{l}\text { Hypocotyl } \\
\text { pubescence }\end{array}$ & Intermediate & Sparse & SPARSE & Resembles male parent \\
\hline 10 & $\begin{array}{l}\text { Anthocyanin on the } \\
\text { nodes }\end{array}$ & Green & Dark purple & Green & Resembles female parents \\
\hline 11 & Stem pubescence & Dense & Sparse & Sparse & Resembles male parent \\
\hline 12 & Plant growth habit & Erect & Erect & Erect & Not determined \\
\hline 13 & Branching habit & Dense & Dense & Dense & Not determined \\
\hline 14 & Leaf density & Dense & Dense & Dense & Not determined \\
\hline 15 & Leaf colour & Green & Dark green & Green & Resembles female parents \\
\hline 16 & Leaf venation & Parallel & Pinnate & Pinnate & Resembles male parent \\
\hline 17 & Leaf type & Compound & Compound & Compound & Not determined \\
\hline 18 & leaf arrangement & Alternate & Alternate & Alternate & Not determined \\
\hline 19 & Leaf pubescence & Sparse & Intermediate & Intermediate & Resembles male parent \\
\hline 20 & $\begin{array}{l}\text { Pedicel position at } \\
\text { anthesis }\end{array}$ & Intermediate & Pendant & Intermediate & Resembles female parents \\
\hline 21 & Filament colour & Green & Purple & Purple & Resembles male parent \\
\hline 22 & Flower position & Intermediate & Pendant & Pendant & Resembles male parent \\
\hline 23 & Fruit shape & Pointed & Sunken & Sunken & Resembles male parent \\
\hline 24 & Fruit surface & Semi-wrinkled & Semi wrinkled & Semi wrinkled & Not determined \\
\hline 25 & $\begin{array}{c}\text { Stigma position at } \\
\text { anthesis }\end{array}$ & Same level & same level & Same level & Not determined \\
\hline 26 & Calyx pigmentation & Present & Present & Present & Not determined \\
\hline 27 & $\begin{array}{l}\text { Fruit colour at } \\
\text { Immature stage }\end{array}$ & Yellow & Green & Green & Resembles male parent \\
\hline 28 & $\begin{array}{c}\text { Fruit colour at } \\
\text { maturity }\end{array}$ & Red & Red & Red & Not determined \\
\hline 29 & Pedicel with fruit & Persistent & Persistent & Persistent & Not determined \\
\hline
\end{tabular}


Ahmodu PB et al. (2020). Not Sci Biol 12(3):658-672

Table 9. Comparison of $\mathrm{R}$ and $\mathrm{S}$

\begin{tabular}{|c|c|c|c|c|c|}
\hline \multicolumn{6}{|c|}{ Determination of dominance between parents } \\
\hline $\begin{array}{l}\mathrm{s} / \\
\mathrm{n}\end{array}$ & Character & Parent 1 & Parent 2 & Hybrid & Inference on mode of inheritance \\
\hline & & $\mathbf{R}$ & $S$ & $\mathrm{RXS}$ & \\
\hline 1 & Stem colour & Green & Purple & Green & Resembles the female parent \\
\hline 2 & Stem pubescence & Intermediate & Sparse & Sparse & Resembles the male parent \\
\hline 3 & Leaf colour & Green & Dark green & Green & Resembles the female parent \\
\hline 4 & Fresh fruit colour & Yellow & Green & Yellow & Resembles the female parent \\
\hline 5 & Fruit pubescence & Sparse & Sparse & Sparse & Not determined \\
\hline 6 & fruit stalk colour & Green & Green & Green & Not determined \\
\hline 7 & Days to germination & 7 & 6 & 7 & Quantitative \\
\hline 8 & Hypocotyl colour & Light green & Purple & Green & Partial dominance \\
\hline 9 & Hypocotyl pubescence & Intermediate & Sparse & Sparse & Resembles the male parent \\
\hline 10 & $\begin{array}{l}\text { Anthocyanin on the } \\
\text { nodes }\end{array}$ & Green & $\begin{array}{c}\text { Dark } \\
\text { purple }\end{array}$ & Green & Resembles the female parent \\
\hline 11 & Stem pubescence & Dense & Dense & Dense & Not determined \\
\hline 12 & Plant growth habit & Erect & Erect & Erect & Not determined \\
\hline 13 & Branching habit & Dense & Dense & Dense & Not determined \\
\hline 14 & Leaf density & Dense & Dense & Dense & Not determined \\
\hline 15 & Leaf colour & Green & Dark green & Green & Resembles the female parent \\
\hline 16 & Leaf venation & Parallel & Pinnate & Parallel & Resembles the female parent \\
\hline 17 & Leaf type & Compound & Compound & Compound & Not determined \\
\hline 18 & leaf arrangement & Alternate & Alternate & Alternate & Not determined \\
\hline 19 & Leaf pubescence & Sparse & Sparse & Sparse & Not determined \\
\hline 20 & $\begin{array}{c}\text { Pedicel position at } \\
\text { anthesis }\end{array}$ & Intermediate & Pendant & Pendant & Resembles the male parent \\
\hline 21 & $\begin{array}{c}\text { Filament colour after } \\
\text { anthesis }\end{array}$ & Green & Purple & Green & Resembles the female parent \\
\hline 22 & Flower position & Intermediate & Pendant & Pendant & Resembles the male parent \\
\hline 23 & Fruit shape & Pointed & Elongated & Pointed & Resembles the female parent \\
\hline 24 & Fruit surface & Semi-wrinkled & $\begin{array}{c}\text { Semi } \\
\text { wrinkled }\end{array}$ & $\begin{array}{c}\text { Semi } \\
\text { wrinkled }\end{array}$ & Not determined \\
\hline 25 & $\begin{array}{c}\text { Stigma position at } \\
\text { anthesis }\end{array}$ & Same level & Exserted & $\begin{array}{l}\text { Same level/ } \\
\text { Excerted }\end{array}$ & No dominance \\
\hline 26 & Calyx pigmentation & Present & Present & Present & Not determined \\
\hline 27 & $\begin{array}{l}\text { Fruit colour at } \\
\text { Immature stage }\end{array}$ & Yellow & Green & Green & Resembles the male parent \\
\hline 28 & Fruit colour at maturity & Red & Red & Red & Not determined \\
\hline 29 & Pedicel with fruit & Persistent & Persistent & Persistent & Not determined \\
\hline
\end{tabular}


Table 10. Comparison of T and $S$

\begin{tabular}{|c|c|c|c|c|c|}
\hline \multicolumn{6}{|c|}{ Determination of dominance between parents } \\
\hline $\mathrm{s} / \mathrm{n}$ & Character & Parent 1 & Parent 2 & Hybrid & Inference on mode of inheritance \\
\hline & & $\mathrm{T}$ & $S$ & $S \times T$ & \\
\hline 1 & Stem colour & Purple & Purple & purple & Not determined \\
\hline 2 & Stem pubescence & Sparse & Sparse & Sparse & Not determined \\
\hline 3 & Leaf colour & Dark green & Dark green & Green & $\begin{array}{c}\text { No dominance nor recessiveness } \\
\text { Leaf colour green }\end{array}$ \\
\hline 4 & Fresh fruit colour & Green & Green & Green & Not determined \\
\hline 5 & Fruit pubescence & Sparse & Sparse & Sparse & Not determined \\
\hline 6 & fruit stalk colour & Green & Green & Green & Not determined \\
\hline 7 & Days to flowering & 6 & 6 & 6 & Quantitative \\
\hline 8 & Hypocotyl colour & Purple & Purple & Purple & Not determined \\
\hline 9 & Hypocotyl pubescence & Sparse & Sparse & Sparse & Not determined \\
\hline 10 & $\begin{array}{l}\text { Anthocyanin on the } \\
\text { nodes }\end{array}$ & Dark purple & Dark purple & purple & No dominance nor recessiveness \\
\hline 11 & Stem pubescence & Sparse & Dense & Dense & Resembles male parent \\
\hline 12 & Plant growth habit & Erect & Erect & Erect & Not determined \\
\hline 13 & Branching habit & Dense & Dense & Dense & Not determined \\
\hline 14 & Leaf density & Dense & Dense & Dense & Not determined \\
\hline 15 & Leaf colour & Dark green & Dark green & Green & No dominance nor recessiveness \\
\hline 16 & Leaf venation & Pinnate & Pinnate & Pinnate & Not determined \\
\hline 17 & Leaf type & Compound & Compound & Compound & Not determined \\
\hline 18 & leaf arrangement & Alternate & Alternate & Alternate & Not determined \\
\hline 19 & Leaf pubescence & Intermediate & Sparse & Sparse & Resembles male parent \\
\hline 20 & $\begin{array}{c}\text { Pedicel position at } \\
\text { anthesis }\end{array}$ & Pendant & Pendant & Pendant & Not determined \\
\hline 21 & Filament colour & Purple & Purple & Purple & Not determined \\
\hline 22 & Flower position & Pendant & Pendant & Pendant & Not determined \\
\hline 23 & Fruit shape & Sunken & Elongated & Sunken & Resembles female parent \\
\hline 24 & Fruit surface & $\begin{array}{c}\text { Semi } \\
\text { wrinkled }\end{array}$ & $\begin{array}{c}\text { Semi } \\
\text { wrinkled }\end{array}$ & Wrinkled & No dominance nor recessiveness \\
\hline 25 & $\begin{array}{l}\text { Stigma position at } \\
\text { anthesis }\end{array}$ & same level & Exserted & Exserted & Resembles male parent \\
\hline 26 & Calyx pigmentation & Present & Present & Present & Not determined \\
\hline 27 & $\begin{array}{c}\text { Fruit colour at immature } \\
\text { stage }\end{array}$ & Green & Green & Green & Not determined \\
\hline 28 & Fruit colour at maturity & Red & Red & Red & Not determined \\
\hline 29 & Pedicel with fruit & Persistent & Persistent & Persistent & Not determined \\
\hline
\end{tabular}

Table 11. Comparison of T and W

\begin{tabular}{|c|c|c|c|c|c|}
\hline \multicolumn{7}{|c|}{ Determination of dominance between parents } \\
\hline s/n & Character & Parent 1 & Parent 2 & Hybrid & Inference on mode of inheritance \\
\hline & & T & W & T X W & \\
\hline 1 & Stem colour & Purple & Green & Green & Resembles male parent \\
\hline 2 & Stem pubescence & Sparse & Dense & Sparse & Resembles female parent \\
\hline 3 & Leaf colour & Dark green & Dark green & Green & No dominance/recessiveness \\
\hline 4 & Fresh fruit colour & Green & Green & Green & Not determined \\
\hline 5 & Fruit pubescense & Sparse & Sparse & Sparse & Not determined \\
\hline 6 & fruit stalk colour & Green & Green & Green & Not determined \\
\hline 7 & Days to flowering & 6 & 6 & 7 & Quantitative \\
\hline 8 & Hypocotyl colour & Purple & White & White & Resembles female parent \\
\hline 9 & Hypocotyl pubescence & Sparse & Intermediate & Sparse & Resembles female parent \\
\hline
\end{tabular}


Ahmodu PB et al. (2020). Not Sci Biol 12(3):658-672

\begin{tabular}{|c|c|c|c|c|c|}
10 & $\begin{array}{c}\text { Anthocyanin on the } \\
\text { nodes }\end{array}$ & Dark purple & Purple & Purple & Resembles male parent \\
\hline 11 & Stem pubescence & Sparse & Dense & Non & No inheritance \\
\hline 12 & Plant growth habit & Erect & Erect & Erect & Not determined \\
\hline 13 & Branching habit & Dense & Dense & Dense & Not determined \\
\hline 14 & Leaf density & Dense & Dense & Dense & Not determined \\
\hline 15 & Leaf colour & Dark green & Green & Green & Resembles male parent \\
\hline 16 & Leaf venation & Pinnate & Parallel & pinnate & Resembles female parent \\
\hline 17 & Leaf type & Compound & Compound & Compound & Not determined \\
\hline 18 & leaf arrangement & Alternate & Alternate & Alternate & Not determined \\
\hline 19 & Leaf pubescence & Intermediate & Intermediate & Intermediate & Not determined \\
\hline 20 & $\begin{array}{c}\text { Pedicel position at } \\
\text { anthesis }\end{array}$ & Pendant & Erect & Erect & Resembles male parent \\
\hline 21 & Filament colour & Purple & Purple & Purple & Not determined \\
\hline 22 & Flower position & Pendant & Erect & Erect & Erect is dominant over pendant \\
\hline 23 & Fruit shape & Sunken & Pointed & Pointed & Resembles male parent \\
\hline 24 & Fruit surface & $\begin{array}{c}\text { Semi } \\
\text { wrinkled }\end{array}$ & Smooth & $\begin{array}{c}\text { Semi } \\
\text { wrinkled }\end{array}$ & Resembles female parent \\
\hline 25 & $\begin{array}{c}\text { Stigma position at } \\
\text { anthesis }\end{array}$ & samelevel & Exserted & Exserted & Resembles male parent \\
\hline 26 & Calyx pigmentation & Present & Present & Present & Not determined \\
\hline 27 & $\begin{array}{c}\text { Fruit colour at } \\
\text { immature stage }\end{array}$ & Green & green/variegated & Green & Resembles female parent \\
\hline 28 & Fruit colour at maturity & Red & Red & Red & Not determined \\
\hline 29 & Pedicel with fruit & Persistent & Persistent & Persitant & Not determined \\
\hline
\end{tabular}

Table 12. Comparison of S and W

\begin{tabular}{|c|c|c|c|c|c|}
\hline \multicolumn{6}{|c|}{ Determination of dominance between parents } \\
\hline $\mathrm{s} / \mathrm{n}$ & Character & Parent 1 & Parent 2 & Hybrid & Inference on mode of inheritance \\
\hline & & $S$ & $\mathrm{~W}$ & $S \mathrm{XW}$ & \\
\hline 1 & Stem colour & Purple & Purple & purple & Not determined \\
\hline 2 & Stem pubescence & Sparse & Sparse & sparse & Not determined \\
\hline 3 & Leaf colour & Dark green & Dark green & Green & No dominance nor recessiveness \\
\hline 4 & Fresh fruit colour & Green & Green & Green & Not determined \\
\hline 5 & Fruit pubescence & Sparse & Non & Non & Resembles the male parent \\
\hline 6 & fruit stalk colour & Green & Green & Green & Not determined \\
\hline 7 & Days to flowering & 6 & 8 & 7 & Quantitative \\
\hline 8 & Hypocotyl colour & Purple & Dark green & Green & Green is dominant over purple \\
\hline 9 & Hypocotyl pubescence & Sparse & Sparse & sparse & Not determined \\
\hline 10 & $\begin{array}{l}\text { Anthocyanin on the } \\
\text { nodes }\end{array}$ & Dark purple & Purple & purple & Not determined \\
\hline 11 & Stem pubescence & Dense & Dense & Dense & Not determined \\
\hline 12 & Plant growth habit & Erect & Erect & Erect & Not determined \\
\hline 13 & Branching habit & Dense & Dense & Dense & Not determined \\
\hline 14 & Leaf density & Dense & Dense & Dense & Not determined \\
\hline 15 & Leaf colour & Dark green & Dark green & Green & Not determined \\
\hline 16 & Leaf venation & Pinnate & Parallel & Pinnate & Resembles female parent \\
\hline 17 & Leaf type & Compound & Compound & Compound & Not determined \\
\hline 18 & leaf arrangement & Alternate & Alternate & Alternate & Not determined \\
\hline 19 & Leaf pubescence & Sparse & Intermediate & Non & No inheritance from either parent \\
\hline 20 & $\begin{array}{c}\text { Pedicel position at } \\
\text { anthesis }\end{array}$ & Pendant & Pendant & Pendant & Not determined \\
\hline 21 & Filament colour & Purple & White & White & Resembles male parent \\
\hline 22 & Flower position & Pendant & Pendant & Pendant & Not determined \\
\hline
\end{tabular}


Ahmodu PB et al. (2020). Not Sci Biol 12(3):658-672

\begin{tabular}{|c|c|c|c|c|c|}
23 & Fruit shape & Elongated & Elongated & Elongated & Not determined \\
\hline 24 & Fruit surface & $\begin{array}{c}\text { Semi } \\
\text { wrinkled }\end{array}$ & Wrinkled & Semi wrinkled & Resembles female parent \\
\hline 25 & $\begin{array}{c}\text { Stigma position at } \\
\text { anthesis }\end{array}$ & Exserted & Exserted & Exserted & Not determined \\
\hline 26 & Calyx pigmentation & Present & Present & Present & Not determined \\
\hline 27 & $\begin{array}{c}\text { Fruit colour at immature } \\
\text { stage }\end{array}$ & Green & Green & Green & Not determined \\
\hline 28 & Fruit colour at maturity & Red & Red & Red & Not determined \\
\hline 29 & Pedicel with fruit & Persistent & Persistent & Persistent & Not determined \\
\hline
\end{tabular}

\section{Discussion}

In this study, closely related members of the genus Capsicum had the highest successful percentage crosses. For example, $\mathrm{T}^{*} \mathrm{~S}, \mathrm{~W}^{*} \mathrm{~T}$ and $\mathrm{S}^{*} \mathrm{~T}$ had the highest percentage success from the crosses carried out; i.e.; 63.33\%, 56.52\% and 54.55\% respectively (being different types of Pepper plants). This implies that intravarietal hybridization is easier in Capsicum spp than inter-varietal hybridization and this agrees with the research of Morakinyo and Falusi (1992).

The high degree of fertilization can be attributed to the high pollen fertility, compatibility between the species and genetic similarities since all the plants are of the same species. This is in accordance with the law of genetic distance between both the species (Geleta et al., 2005).

The species with the lowest hybridization are $T^{*} \mathrm{R}$ which had a successful fertilization of $18.97 \%$. $\mathrm{R}^{*} \mathrm{~S}$ had a percentage fertilization of $25.53 \%, \mathrm{R}^{*} \mathrm{~T}$ had a percentage fertilization of $27.45 \%$ while $\mathrm{W}^{*} \mathrm{~S}$ had a percentage fertilization of $30.30 \%$. The low percentage of fertilization could be as a result of genetic incompatibility, failure of the pollen tube to germinate on the stigma. Failure of the pollen tube to germinate to reach the tube or abortion of same (Zhang, 2010).

There was no hybridization with zero percentage cross success. This further enforces how closely related and compatible species of Pepper are. In the study carried out by Morakinyo and Falusi (1992), on the chromosome behavior of some selected species of Capsicum, this was also discovered, and it enforces how closely related species of Pepper are due to the chromosomal behavior.

Genetic exchange of characters was noticed during the crosses. Gene exchange between groups or individual organisms can be influenced by several factors which may include physical barriers against cross pollination. Such barriers are overcome through artificial land pollination. However, there are other postpollination barriers which artificial land pollination cannot remove. In all cases the effectiveness of these barriers depends on the genetic relationship between individuals and groups. Personal success of artificial land pollination is an indicator of this genetic relatedness and possibility of the crosses occurring in the wild. This accounts for the numerous varieties of Pepper in cultivation all around the world. This was ascertained by Morakinyo and Falusi (1992). The higher the percentage success from the crosses carried out, the higher the genetic relatedness or the closer the genetic relationship of the individuals (Falusi and Morakinyo, 1994).

Pathogenesis was also discovered in some $\mathrm{F}_{1}$ fruits as they developed without seeds in them. Some seeds gotten from the $F_{1}$ generation were not viable. This led to a reduction in the number of $F_{1}$ plants. This is in correlation with the work of Hundal and Dhall (2005).

From the Quantitative data of hybrids and reciprocals studied in the research. The hybrids and reciprocals exhibited segregation of characters in the $F_{1}$ and independent assortment of characters in $F_{2 .} 9$ quantitative characters were studied and mean values were analyzed using analysis of variance (ANNOVA) and the Duncan of plant height (PH), leaf length (LL), leaf breadth (LB), stem girth (SG), laminar leaf length $(\mathrm{LLL})$, anthal length $(\mathrm{AL})$, style length $(\mathrm{SL})$, petal length $(\mathrm{PL})$ and petal width $(\mathrm{PW})$. 
The variation in $\mathrm{PH}$ between the varieties a hybrid show that the different varieties of pepper have varying gene alleles for the character. There was drastic increase in variation of $\mathrm{PH}$ in the hybrid $\mathrm{R}^{*} \mathrm{~W}_{2}$ (36.20 \pm 4.39$)$ which is an increase from the hybrid parents $\mathrm{R}(27.22 \pm 3.62)$ and $\mathrm{W}(39.30 \pm 2.21)$. There was a decrease in the $\mathrm{PH}$ of hybrid $S^{*} \mathrm{~W}(35.86 \pm 0.80)$ compared to the variation in $\mathrm{PH}$ of parent plants $S$ $(35.20 \pm 1.48)$ and $W(39.30 \pm 2.21)$.

There was variation in LL among the hybrids. Notably in $\mathrm{W}^{*} \mathrm{R}(7.80 \pm 0.29)$ relative the parent plants $\mathrm{W}(12.64 \pm 0.92)$ and $\mathrm{R}(17.14 \pm 0.71)$. This was also noted in $S^{*} \mathrm{~T}(8.06 \pm 0.31)$ from the parents $S(13.30 \pm 0.45)$ and $\mathrm{T}(15.30 \pm 0.45)$. This can be attributed to variation in alleles of the genes responsible for LL between parents. No dominance nor recessiveness of this trait among the parents.

With hybridization there was an increase in $S G$ in the hybrid offspring of the cross. $T^{*} R, T^{*} W, W^{*} S$ and $S^{*} T$ had drastic increase in SG. There was variation of LLL among hybrid plants and their parents. This can be attributed to the random pairing of the alleles of the genes that regulate these characters.

Majorities of the data followed maternal inheritance. This can be found in Tables 2 to 10 . While few amongst the characters followed paternal inheritance. Majority of the characters could not be determined since both parents showed similar attributes for the same character. Examples of these characters are; calyx pigmentation, leaf type, leaf venation, leaf arrangement, e.t.c.

From Figure 2, it was noticed that hybrid fruits are smaller in size in size when compared to their parent fruits. About $70 \%$ of the fruits were discovered to have shrinked especially when crossed with species of smaller sizes.

Based on interspecific crosses, it was seen that crosses between $C$. frutasense ${ }^{*} C$. annuum are higher at an average percentage than crosses between $C$. annuum and $C$. frutasence. Both at an average percentage. This means reciprocal successes are few.

Most plants crossed with $\mathrm{W}$ at their orientation of flower and fruits showed alternation of the character of flower orientation as some plants had either erect flowers like W while others had pendant flowers. Plants crossed with Whad similar erect flowers which were not shown in W parent plants and this implies that erect flower orientation could be said to be coded by a heterogenous pair of chromosomes. This is found in table 15 . Similar result was observed in the work done by Nwankiti (1976) and Odland and Porter (1941).

\section{Conclusions}

Based on these results, it can be concluded crosses between varieties of the same species (intervarietal crosses) are more successful than interspecific crosses. There are minimum successes of interspecific crosses among the genus Capsicum in Nigeria. Furthermore, characters follow maternal inheritance than paternal inheritance. Therefore, there is natural gene exchange. This could be used as a baseline for the genetic improvement of Capsicum plants.

\section{Authors' Contributions}

Methodology: JAM and CTA, Supervision: JAM, Writing - review and editing: JAM, OTM. Project administration: KSO. All authors read and approved the final manuscript.

\section{Acknowledgements}

This research received no specific grant from any funding agency in the public, commercial, or not-forprofit sectors. 


\section{Conflict of Interests}

The authors declare that there are no conflicts of interest related to this article.

\section{References}

Bosland PW, Votava EJ (2000). Peppers: vegetable and spice Capsicums. CAB International, England, pp 233. https://doi.org/10.1080/01140671.2012.745161

Falusi OA, Morakinyo JA (1994). Intra and inter specific hybridization in the genus Capsicum. African Crop Science Journal 2(2):169-171. https://doi.org/10.4314/acsj.v2i2.54703

Geleta LF, Labuschagne MT, Viljoen CD (2005). Genetic variability in pepper (Capsicum annuum L.) estimated by morphological data and amplified fragment length polymorphism markers. Biodiversity and Conservation 14:2361-2375. https://doiorg/10.1007/s10531-004-1669-9

Hundal JS, Dhall RK (2005). Breeding for hybrid hot pepper. Journal of New Seeds 2(2):1-22. https://doi.org/10.1300/J153v06n02_03

Nwankiti OC (1976). Cytogenetics of some Nigerian peppers - genus Capsicum. II. Hybrid origin of C. frutescens. Nigerian Journal of Science 10:61-75.

Odland ML, Porter AM (1941). A study of natural crossing in peppers (Capsicum frutescens). Proceedings of the American Society for Horticultural Science 38:585-588.

Reilly CA, Crouch DJ, Yost GS (2001). Quantitative analysis of capsaicinoids in fresh peppers, oleoresin capsicum and pepper spray products. Journal of Forensic Sciences 46:502-509.

Singh RJ (2002). Plant cytogenetics. CRC Press, London, pp 488.

Singh RJ (2002). Genetic resources, chromosome engineering and crop improvement: vegetable crops. Volume 3, CRC Press.

Zhang BX, Wang LH, Mao SL, Zhang ZH (2010). Research progress on pepper breeding and genetic during China's Eleventh Five-year Plan. China Vegetables 24:1-9.
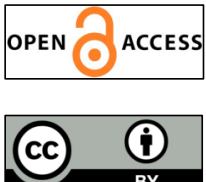

The journal offers free, immediate, and unrestricted access to peer-reviewed research and scholarly work. Users are allowed to read, download, copy, distribute, print, search, or link to the full texts of the articles, or use them for any other lawful purpose, without asking prior permission from the publisher or the author.

License - Articles published in Notulae Scientia Biologicae are Open-Access, distributed under the terms and conditions of the Creative Commons Attribution (CC BY 4.0) License.

(c) Articles by the authors; SHST, Cluj-Napoca, Romania. The journal allows the author(s) to hold the copyright/to retain publishing rights without restriction. 